\title{
Jacobi Elliptic Function Expansion Method for the Nonlinear Vakhnenko Equation
}

\author{
Chunhuan Xiang1, Honglei Wang2* \\ ${ }^{1}$ School of Public Health and Management, Chongqing Medical University, Chongqing, China \\ ${ }^{2}$ College of Medical Informatics, Chongqing Medical University, Chongqing, China \\ Email: w8259300@126.com, *w8259300@163.com
}

How to cite this paper: Xiang, C.H. and Wang, H.L. (2020) Jacobi Elliptic Function Expansion Method for the Nonlinear Vakhnenko Equation. Journal of Applied Mathematics and Physics, 8, 793-798. https://doi.org/10.4236/jamp.2020.85061

Received: April 7, 2020

Accepted: April 27, 2020

Published: April 30, 2020

Copyright $\odot 2020$ by author(s) and Scientific Research Publishing Inc. This work is licensed under the Creative Commons Attribution International License (CC BY 4.0).

http://creativecommons.org/licenses/by/4.0/ (c) (i) Open Access

\begin{abstract}
By using Jacobi elliptic function expansion method, several kinds of travelling wave solutions of Nonlinear Vakhnenko equation are obtained in this paper. As a result, some new forms of traveling wave solutions of the equation are shown, and the numerical simulation with different parameters for the new forms solutions are given.
\end{abstract}

\section{Keywords}

Jacobi Elliptic Function, Expansion Method, Nonlinear Vakhnenko Equation, Solitary Solution, Travelling Wave Solutions

\section{Introduction}

The nature of the world is known by people step by step with many powerful methods. Solving linear equation is the beginning of the process, meanwhile, the results do not well agree with the solution of the linear equation. People overcome the difference and find the nonlinear equation, which is used to describe many phenomena in various physics fields. Many different nonlinear equations appear gradually. Nonlinear Vakhnenko equation is a kind of nonlinear partial differential equation, which is proposed to describe long waves of small amplitudes broadcasting in nonlinear dispersive media. The equation and its variable coefficients play an important role in further study mathematics and knowing the physics of nature. However, obtaining the solutions of the nonlinear equation is a difficult story. With deeply studying the relation of nature, many powerful methods appear [1]-[7]. The nonlinear Vakhnenko equation is investigated in many papers [8] [9] [10] [11]; some new solutions in the form of Jacobi elliptic function are given in this paper, which enrich the kinds of the solution 
for the equation.

Generally, the nonlinear Vakhnenko equation [12] is read as

$$
u u_{x x t}-u_{x} u_{x t}+u^{2} u_{t}=0
$$

where the subscripts denote the partial derivatives of $x$ and $t$. The remaining structure of this paper is organized as follows: Section 2 is a brief introduction to the Jacobi elliptic function and its properties. In Section 3, by implementing the Jacobi elliptic function expansion method, some new traveling wave solutions for nonlinear Vakhnenko are reported. The conclusion is summarized in the last section.

\section{The Jacobi Elliptic Function Expansion Method and Its Properties}

The nonlinear partial differential equation with independent variables $x$ and $t$ is generally in the following form

$$
Q\left(u, u_{x}, u_{t}, u_{x t}, u_{x x}, u_{t t}, \cdots\right)=0
$$

The above equation is a function about $u(x, t)$, the subscripts denote the partial derivatives with $x$ and $t$, respectively. The wave variable $\xi=x+w t$ is applied to the Equation (2), which is changed into the following ordinary differential equation

$$
Q\left(u, u_{\xi}, u_{\xi \xi}, u_{\xi \xi \xi}, \cdots\right)=0
$$

where $u_{\xi}, u_{\xi \xi}, \cdots$ denotes the derivative with respect to the same variable $\xi$.

Generally, the function $u$ in terms of Jacobi elliptic function expansion method can be expressed with the first kind of Jacobi elliptic function $\operatorname{sn}(\xi)$ as follow

$$
u(\xi)=\sum_{i}^{m} a_{i} s n^{i}(\xi)
$$

where $a_{i}$ is constant parameter; $m$ is determined by balancing the linear term of the highest order derivative with nonlinear term of Equation (3).

The three kinds of Jacobi elliptic functions have useful properties [12] [13] [14] [15] [16], which are given as follow:

$$
\begin{gathered}
\operatorname{sn}(\xi)^{2}=1-\operatorname{cn}(\xi)^{2}, d n(\xi)^{2}=1-m^{2} \operatorname{sn}(\xi)^{2} \\
\operatorname{sn}(\xi)_{\xi}=\operatorname{cn}(\xi) d n(\xi), \operatorname{cn}(\xi)_{\xi}=-\operatorname{sn}(\xi) d n(\xi), \\
d n(\xi)_{\xi}=-m^{2} \operatorname{sn}(\xi) \operatorname{cn}(\xi)
\end{gathered}
$$

where $m(0<m<1)$ is the modulus of the elliptic function. The modulus substantially affects the Jacobi elliptic solutions, which will asymptotically go into hyperbolic functions and trigonometric functions when the modulus $m \rightarrow 1$ and $m \rightarrow 0$, respectively. The asymptotical functions are listed in the following

$$
\begin{aligned}
& m \rightarrow 1, \operatorname{sn}(\xi) \rightarrow \tanh (\xi), \operatorname{cn}(\xi) \rightarrow \operatorname{sech}(\xi), d n(\xi) \rightarrow \operatorname{sech}(\xi) \\
& m \rightarrow 0, \operatorname{sn}(\xi) \rightarrow \sin (\xi), \operatorname{cn}(\xi) \rightarrow \cos (\xi), d n(\xi) \rightarrow 1 .
\end{aligned}
$$




\section{Jacobi Elliptic Function Expansion Method for Nonlinear Vakhnenko Equation}

Consider the Nonlinear Vakhnenko equation in the form of Equation (1) with $\xi=x+w t$, we obtain

$$
u u_{\xi \xi \xi}-u_{\xi} u_{\xi \xi}+u^{2} u_{\xi}=0
$$

By using $m$ to balance the highest order derivative term and the nonlinear term of Equation (9), we have $m=2$ obtained from Equation (9). Then, Equation (4) reduced as

$$
u(\xi)=a_{0}+a_{1} s n(\xi)+a_{2} s n^{2}(\xi)
$$

where $s n(\xi)$ is the first kind of Jacobi elliptic function.

Substituting Equation (10) into the equation Equation (9) and collecting all terms with the same power term together and equating each coefficient of $\operatorname{sn}(\xi)$ to zero, a set of simultaneous algebraic equations are yielded as follows:

$$
\begin{gathered}
-a_{0} a_{1}+a_{0}^{2} a_{1}-2 a_{1} a_{2}-a_{0} a_{1} m^{2}=0 \\
2 a_{1}^{2} a_{0}-8 a_{0} a_{2}+2 a_{0}^{2} a_{2}-4 a_{2}^{2}-8 a_{2} a_{0} m^{2}=0 \\
a_{1}^{3}-3 a_{1} a_{2}+6 a_{0} a_{1} a_{2}+6 a_{0} a_{1} m^{2}-3 a_{1} a_{2} m^{2}=0 \\
4 a_{1}^{2} a_{2}+4 a_{2}^{2} a_{0}+4 a_{1}^{2} m^{2}+24 a_{0} a_{2} m^{2}=0 \\
5 a_{2}^{2} a_{1}+20 a_{2} a_{1} m^{2}=0 \\
2 a_{2}^{3}+12 a_{2}^{2} m^{2}=0
\end{gathered}
$$

Solving the above algebraic equations, we get the results:

$$
a_{0}=2\left(m^{2}+1\right) \pm 2 \sqrt{m^{4}-m^{2}+1} ; \quad a_{1}=0 ; \quad a_{2}=-6 m^{2}
$$

By using Equation (10), we obtained the solution of Equation (1) as the following:

$$
\begin{aligned}
& u_{1}(\xi)=2\left(m^{2}+1\right)+2 \sqrt{m^{4}-m^{2}+1}-6 m^{2} s^{2}(\xi) \\
& u_{2}(\xi)=2\left(m^{2}+1\right)-2 \sqrt{m^{4}-m^{2}+1}-6 m^{2} \operatorname{sn}^{2}(\xi)
\end{aligned}
$$

where $\xi=x+w t$.

The numerical simulation for Equations ((17), (18)) with $m=0.3, w=0.5$, $x \in[-10,10]$ and $t \in[-10,10]$ are shown in Figure 1 and Figure 2, respectively. The values of the parameters here and the following are determined randomly in the corresponding interval.

According to the asymptotically functions, the numerical simulation for Equation (18) with $w=0.5, x \in[-10,10], t \in[-10,10], \quad m=0.99999999$ and $m=0.00000001$, are shown in Figure 3 and Figure 4, respectively. The Equation (18) is changed into hyperbolic function and trigonometric function when the modulus approaches $1(m \rightarrow 1)$ and $0(m \rightarrow 0)$, which are listed as follows

$$
\begin{aligned}
& u_{3}(\xi)=2\left(m^{2}+1\right)-2 \sqrt{m^{4}-m^{2}+1}-6 m^{2} \tanh ^{2}(\xi) \\
& u_{4}(\xi)=2\left(m^{2}+1\right)-2 \sqrt{m^{4}-m^{2}+1}-6 m^{2} \sin ^{2}(\xi)
\end{aligned}
$$


The derivative of the Equation (18) is given as

$$
u_{5}(\xi)=-12 m^{2} \operatorname{sn}(\xi) c n(\xi) d n(\xi) .
$$

The numerical simulation with $m=0.3, w=0.5, \quad x \in[-10,10]$ and $t \in[-10,10]$ is shown in Figure 5 .

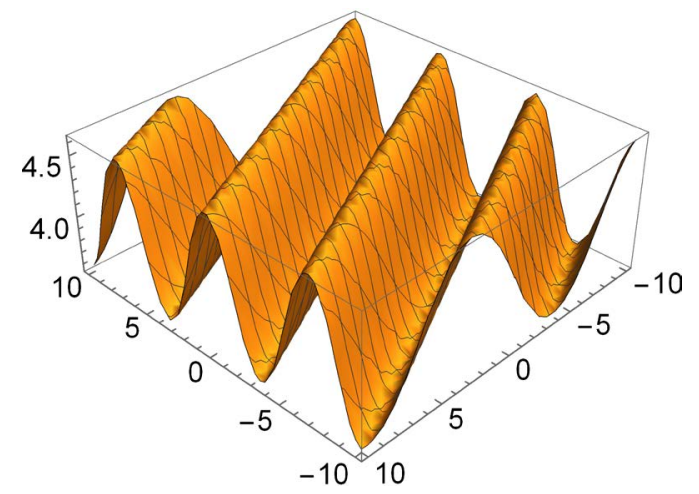

Figure 1. The numerical simulation for Equation (17) with $m=0.3, w=0.5, \quad x \in[-10,10]$ and $t \in[-10,10]$.

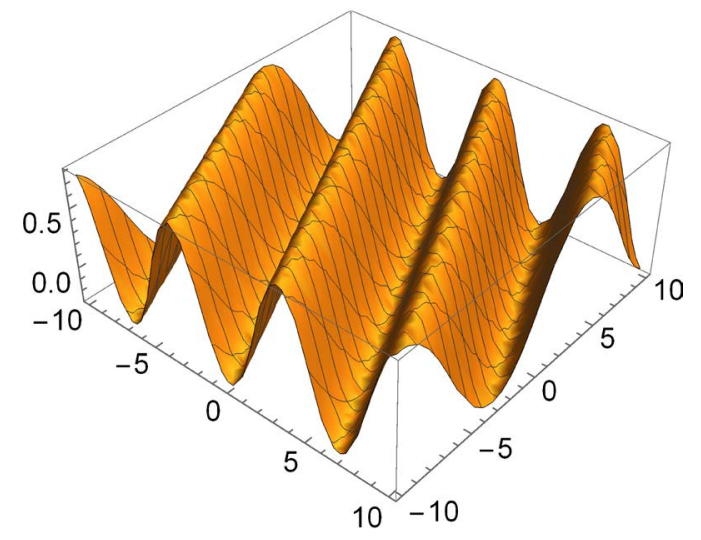

Figure 2. The numerical simulation for Equation (18) with $m=0.3, \quad w=0.5, \quad x \in[-10,10]$ and $t \in[-10,10]$.

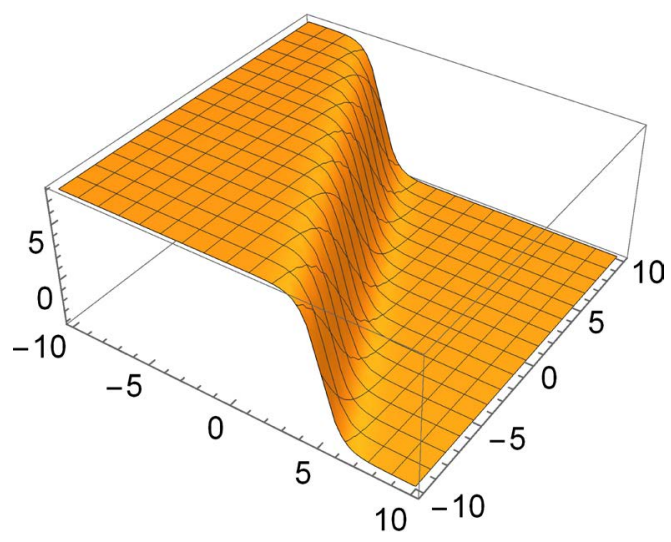

Figure 3. The numerical simulation for Equation (18) with $m=0.99999999, w=0.5$, $x \in[-10,10]$ and $t \in[-10,10]$. 


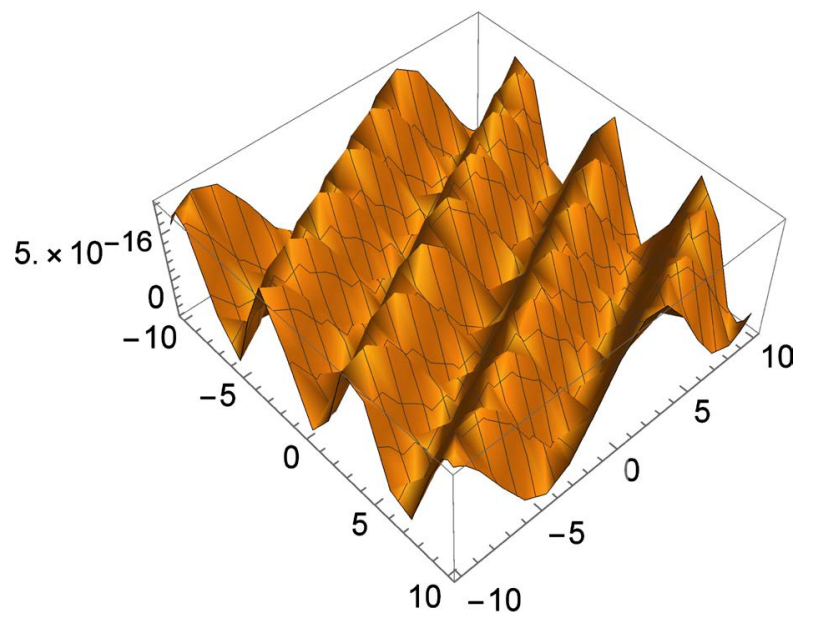

Figure 4. The numerical simulation for Equation (18) with $m=0.00000001, w=0.5$, $x \in[-10,10]$ and $t \in[-10,10]$.

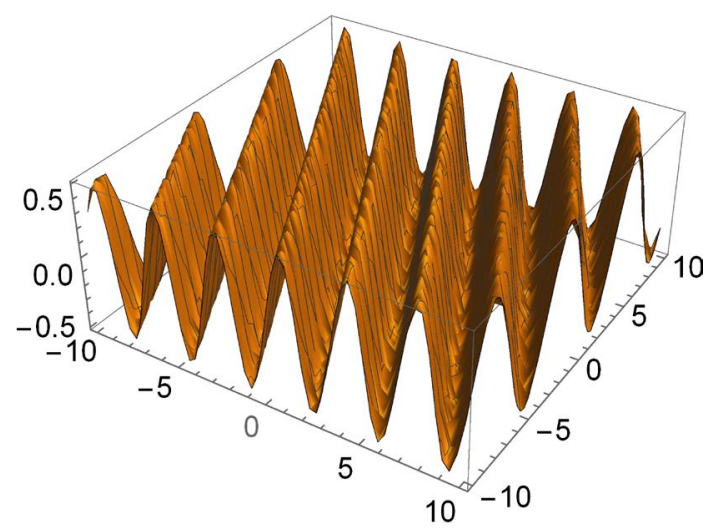

Figure 5. The numerical simulation for Equation (21) with $m=0.3, w=0.5, \quad x \in[-10,10]$ and $t \in[-10,10]$.

\section{Conclusion}

The nonlinear Vakhnenko equation in the form Equation (1) has been further studied by means of the Jacobi elliptic function expansion method. Several kinds of periodic evolution solutions (17)-(21) of Equation (1) are obtained in terms of Jacobi elliptic functions, hyperbolic solutions and trigonometric solutions. Those solutions can be used to discuss some interesting physical phenomena of amplitudes broadcasting in nonlinear dispersive media. The performance of Jacobi elliptic functions expansion method is powerful, which gives more solutions. From the simulation results, we can know the amplitudes of travelling wave solutions and period. Equation (21) is the derivative of the Equation (18), which shows the velocity of the wave solutions.

\section{Conflicts of Interest}

The authors declare no conflicts of interest regarding the publication of this paper. 


\section{References}

[1] Zhu, Z.N. (1992) Soliton-Like Solutions of Generalized KdV Equation with External Force Term. Acta Hysica Sinca, 41, 1561-1566.

[2] Zhang, J.F. and Chen, F.Y. (2001) Truncated Expansion Method and New Exact Soliton-Like Solution of the General Variable Coeffcient KdV Equation. Acta Physica Sinca, 50, 1648-1650.

[3] Malfliet, M. and Wieers, E. (1996) A Nonlinear Theory of Charged-Particle Stopping in Non-Ideal Plasmas. Journal of Plasma Physics, 56, 441-443. https://doi.org/10.1017/S0022377800019401

[4] Wang, Y. and Wang, B. (2005) High-Order Multi-Symplectic Schemes for the Nonlinear Klein-Gordon Equation. Applied Mathematics and Computation, 166, 608-632. https://doi.org/10.1016/j.amc.2004.07.007

[5] Liu, S.K., Fu, Z.T. and Liu, S.D. (2001) A Simple Fast Method in Finding Particular Solutions of Some Nonlinear PDE. Applied Mathematics and Mechanics, 22, 326-331. https://doi.org/10.1007/BF02437971

[6] Vu-Quoc, L. and Li, S. (1993) Invariant-Conserving Finite Difference Algorithms for the Nonlinear Klein-Gordon Equation. Computer Methods in Applied Mechanics and Engineering, 107, 341-391. https://doi.org/10.1016/0045-7825(93)90073-7

[7] Bonanno, C. (2010) Existence and Multiplicity of Stable Bound States for the Nonlinear Klein-Gordon Equation. Nonlinear Analysis, 72, 2031-2046. https://doi.org/10.1016/j.na.2009.10.004

[8] Yusufolu, E. and Bekir, A. (2008) The Tanh and the Sine-Cosine Methods for Exact Solutions of the MBBM and the Vakhnenko Equations. Chaos Solitons \& Fractals, 38, 1126-1133. https://doi.org/10.1016/j.chaos.2007.02.004

[9] Mo, J.-Q. (2011) Travelling Wave Solution of Disturbed Vakhnenko Equation for Physical Model. Acta Physica Sinica, 60, Article ID: 090203.

[10] Parkes, E.J. (1993) The Stability of Solutions of Vakhnenko's Equation. Journal of Physics: A General Physics, 26, 6469. https://doi.org/10.1088/0305-4470/26/22/040

[11] Morrison, A.J. and Parkes, E.J. (2001) The N-Soliton Solution of a Generalised Vakhnenko Equation. Glasgow Mathematical Journal, 43, 65-90. https://doi.org/10.1017/S0017089501000076

[12] Liu, G.-T. and Fan, T.-Y. (2004) Jacobi Elliptic Function Expansion Method under a General Function Transform and Its Application. Acta Physica Sinca, 53, 676-679.

[13] Prasolov, V. and Solovyev, Y. (1997) Elliptic Functions and Elliptic Integrals. American Mathematical Society, Providence.

[14] Chamdrasekharan, K. (1985) Elliptic Functions. Springer-Verlag, Berlin.

[15] Liu, G.-T. (2009) Lame Equation and Asymptotic Higher-Order Periodic Solutions to Nonlinear Evolution Equations. Applied Mathematics and Computation, 212, 312-317. https://doi.org/10.1016/j.amc.2009.02.022

[16] Liu, L. (2010) WITHDRAWN: Exact Travelling Wave Solutions for the Nizhnik-Novikov-Veselov Equation. Applied Mathematics and Computation. 